\title{
Efficacy of a Homeopathic Medicine of Capsicum frutescens L. (Solanaceae) in the Treatment of Hot Flashes in Menopausal Women: A Phase-2 Randomized Controlled Trial
} \author{
Edson Zangiacomi Martinez ${ }^{2}$ Ana Maria Soares Pereira ${ }^{3}$ \\ ${ }^{1}$ Department of Pediatrics, Ribeirão Preto Medical School, University \\ of São Paulo (USP), São Paulo, Brazil \\ 2 Department of Social Medicine, Ribeirão Preto Medical School, \\ University of São Paulo (USP), São Paulo, Brazil \\ ${ }^{3}$ Department of Plant Biotechnology, University of Ribeirão Preto \\ (UNAERP), Ribeirão Preto, Brazil
}

Débora Cristiane da Silva Andrade ${ }^{1}$ Fabio Carmona ${ }^{1}$ Mateus Andrea Angelucci ${ }^{1}$

\begin{abstract}
Address for correspondence Fabio Carmona, MD, PhD, Department of Pediatrics, Ribeirão Preto Medical School, University of São Paulo, Avenida dos Bandeirantes S/N, Campus Universitario, Ribeirão Preto, SP, Brazil 14049-900 (e-mail: carmona@fmrp.usp.br).
\end{abstract}

Homeopathy 2019;108:102-107.

\begin{abstract}
Background Hot flashes are common in women during menopause, and are an important cause of discomfort, increasing the number of medical appointments. Hormone replacement therapy is an effective treatment, but it can bring undesirable consequences. Alternative treatments exist but they are not universally accepted or effective. The ingestion of malagueta peppers (popular name for fruits of Capsicum frutescens L., Solanaceae) causes sensations similar to those experienced by women during hot flashes. Using the homeopathic law of similars (let like be cured by like), we hypothesized that a homeopathic remedy made of malagueta peppers can be effective in alleviating menopausal hot flashes. We named this remedy Malagueta.

Methods This randomized, placebo-controlled, double-blind, phase-2 clinical trial was designed to test the hypothesis that, in menopausal women, the homeopathic medicine Malagueta $(30 \mathrm{CH})$, compared with placebo, will significantly reduce the intensity of hot flashes, after 4 weeks of treatment. The primary outcome was the intensity of hot flashes, measured by the Measure Yourself Medical Outcome Profile (MYMOP) instrument. A total of 40 women were enrolled in the study, 20 in each group. Results The effect of Malagueta on the primary outcome, the intensity of hot flashes, assessed by MYMOP, was superior to that of placebo over the 4 weeks of treatment, with worsening in both groups after treatment was interrupted (after week 4, $p<0.001$ in ordinal logistic regression). The odds ratio for treatment response

\section{Keywords}

- menopause

- hot flashes

- pepper

- Solanaceae

- homeopathy (reduction of at least three MYMOP categories) was 2.78 (95\% confidence interval, 0.77 to 10.05 ). Treatment with Malagueta, compared with placebo, also reduced the intensity of the secondary symptoms $(p=0.001)$ and improved level of activity $(p=0.025)$ and well-being $(p=0.008)$.

Conclusion The homeopathic medicine of Capsicum frutescens (Malagueta) was superior to placebo in reducing the intensity of hot flashes in menopausal women after 4 weeks of treatment.
\end{abstract}

received

July 28, 2018

accepted after revision

October 14, 2018

published online

February 8, 2019
Copyright @ 2019 The Faculty of Homeopathy
DOI https://doi.org/ $10.1055 / \mathrm{s}-0038-1676326$. ISSN $1475-4916$ 


\section{Introduction}

Menopausal hot flashes are sudden, transient feelings of heat and sweat, predominantly in the upper part of the body. ${ }^{1}$ They are a common symptom in menopausal women, with a variable incidence worldwide (18-74\%), ${ }^{2}$ leading women to seek relief in medical appointments and use of supplements. Hot flashes can be precipitated by stress, heat, alcohol, caffeine, and spicy food.

Though the pathophysiology of menopausal hot flashes is not understood, treatment has been carried out with hormone replacement therapy. ${ }^{1}$ However, the risk of burden with hormones has led many women to look for alternative therapies. ${ }^{1,3}$

Non-hormonal therapies for menopausal hot flashes include clonidine, selective serotonin (SSRIs) or serotoninnorepinephrine (SNRIs) reuptake inhibitors gabapentin, and others, all leading to mild-to-moderate side-effects in women with a history of breast cancer. ${ }^{4}$

Among the alternative therapies, herbal medicines are widely used, including plants such as soy (Glycine max), black cohosh (Actaea racemosa), dong quai (Angelica sinensis), hops (Humulus lupulus), Mexican yam (Dioscorea villosa), ginseng (Panax ginseng), evening primrose oil (Oenothera biennis), Saint John's wort (Hypericum perforatum), ginkgo (Ginkgo biloba), and others. Besides herbal medicines, many women experience relief with relaxation techniques, acupuncture, and homeopathy. ${ }^{4}$ Three homeopathic medicines are commonly used for menopausal symptoms: Lachesis (produced from the venom of Lachesis genera), Pulsatilla (obtained from the plant Anemone pulsatilla), and Sepia (produced from ink of cuttlefish [Sepiida order, Cephalopoda class]). Nevertheless, none of these therapies is universally accepted or effective. ${ }^{4}$

Capsicum frutescens L. (Solanaceae, synonym Capsicum annuum var. frutescens (L.) Kuntze) comprises a wide group of red, hot peppers native to the Americas. ${ }^{5}$ One variety of red pepper is known as malagueta in Brazil. When people eat malagueta pepper, they experience burning-like sensations in the mouth, tachycardia, sialorrhea, rhinorrhea and, interestingly, mild-to-intense sensations of warmth and sweat, mostly in face and neck. ${ }^{6}$

Therefore, considering the similarity between the sensations caused by eating malagueta peppers and those of menopausal hot flashes, and considering the homeopathic principle of similia similibus curantur, that is, the law of similars, we hypothesized that a homeopathic remedy made of malagueta peppers can be effective in alleviating menopausal hot flashes. We named this remedy Malagueta.

This study was designed to test the hypothesis that, in menopausal women, the homeopathic medicine of malagueta pepper, compared with placebo, will significantly reduce the intensity of hot flashes, after 4 weeks of treatment.

\section{Patients and Methods}

This was a randomized, double-blind, placebo-controlled, phase-2 clinical trial on clinical homeopathy (clinical, formula, single constituent). This study was approved by a local institutional review board (Research Ethics Committee, Clinics Hospital, Ribeirão Preto Medical School, University of São Paulo [USP]), under protocol no. 2806/2011. Informed consent was obtained from all participants. The study was also registered in ClinicalTrials.gov (protocol no. NCT 01315041). This study adheres to CONSORT $^{7}$ (- Supplementary Table 1, available in online version only) and RedHot ${ }^{8}$ statements (-Supplementary Table 2 , available in online version only).

The participants were recruited at a single gynecologist's office. Briefly, the setting was a private outpatient clinic with six offices ( 3 physicians, 1 dentist, 1 psychologist, and 1 nutritionist). The physician's office was a $25 \mathrm{~m}^{2}$-room with a private bathroom. The gynecologist saw an average of 30 patients per week. All menopausal women within STRAW +10 categories -1 to +1 c were potentially eligible for the study. ${ }^{9}$ Inclusion criteria were woman with menopausal hot flashes as major complaint, not having taken part in another clinical trial within the previous 6 months, and not receiving any treatment for hot flashes or menopause, including anti-depressants. Overall, since participants were recruited in a private clinic, they had good socioeconomic and educational status. Hot flashes were defined as sudden and transient feelings of heat and sweat in the upper body, including chest, neck and face, that occur during menopause. Women were not included if they had any previous relationship with the researchers, history of hypersensitivity or dermatitis due to any kind of pepper, or hemorrhoids. Exclusion criteria were the development of any adverse event, of any severity, that could be attributed to the medicine (in this case with unblinding), or at a participant's request. Adverse events were monitored by weekly phone calls from the attending physician. In the case of any serious adverse event, the patient would have been fully assisted by the attending physician and the research team. Toxicity was extremely unlikely because a potency of $30 \mathrm{CH}$ overcomes the Avogadro limit.

\section{Preparation of Medicine and Placebo}

The species $C$. frutescens was grown and harvested at the rural area of Jardinópolis, São Paulo, Brazil (latitude $21^{\circ} 4^{\prime} 33^{\prime \prime}$ $\mathrm{S}$, longitude $47^{\circ} 44^{\prime} 48^{\prime \prime} \mathrm{W}$ ) with authorization from the Brazilian government. A specimen was deposited at the Herbarium of the University of Ribeirão Preto (UNAERP), with voucher number HPMU 3216. The ripe fruits (i.e., the peppers) were collected and dried in a circulating-air oven $\left(45^{\circ} \mathrm{C}\right)$ for 3 days. They were powdered in a knife-mill and extracted with EtOH $/ \mathrm{H}_{2} \mathrm{O}(70 \% \mathrm{w} / \mathrm{v})$ in a Soxhlet extractor for 8 hours, resulting in the mother tincture. In the first three centesimal dynamizations, $\mathrm{EtOH} / \mathrm{H}_{2} \mathrm{O}(70 \% \mathrm{w} / \mathrm{v})$ was used as vehicle, according to the Brazilian Pharmacopeia. ${ }^{10}$ Briefly, 1 drop of the crude extract was diluted in 99 drops of the vehicle, and the mixture was mechanically agitated 100 times, resulting in the first centesimal dilution $(1 \mathrm{CH})$. The procedure was repeated up to a $30 \mathrm{CH}$ solution. The vehicle for the last dilution was $\mathrm{EtOH} / \mathrm{H}_{2} \mathrm{O}(30 \% \mathrm{w} / \mathrm{v})$. Placebo was an $\mathrm{EtOH} / \mathrm{H}_{2} \mathrm{O}(30 \% \mathrm{w} / \mathrm{v})$ solution without agitation.

\section{Protocol}

All patients seen during the recruitment phase were screened for eligibility according to the above criteria. The potentially eligible women underwent a classical medical interview, focusing on hot flashes as their major complaint and, upon 
fulfilling the inclusion criteria, they were invited to enrol in the study. Details on symptoms were restricted to those in the research questionnaires. The physician made sure the patient understood every aspect of participating in a clinical trial, including the responsibilities of both parties. After consenting, they received medicine or placebo, at no cost, in sufficient quantity for 4 weeks. Subsequent contacts were made by phone calls up to the final patient visit at week 8 .

The investigators were experienced in prescribing homeopathy and in clinical research. Briefly, they comprised five individuals: (1) DCSA: MD, MSc, physician (gynecologist), responsible for recruitment, clinical assessment, medicine prescription, and follow-up, with little experience in homeopathy, blinded to allocation; (2) FC: MD, PhD, physician (pediatrician), responsible for study design, randomization, data analysis, and manuscript preparation, with little experience in homeopathy, not blinded to allocation, experienced in clinical research; (3) MAA: MD, MSc, physician (psychiatrist), responsible for study rationale and design, with 18 months of formal training in homeopathy (classical style) at Lamasson Institute (https://www.lamasson.com.br), Ribeirão Preto, SP, Brazil, followed by 15 years of experience in prescription of homeopathic and herbal medicines in a general, beneficent outpatient clinic, and in private practice, blinded to allocation; (4) EZM: PhD, statistician, responsible for study design, data quality and analysis, with some experience in homeopathy, blinded to allocation, experienced in clinical research; and (5) AMSP: PhD, chemist, responsible for study design, preparation of medicine and placebo, with some experience in homeopathy, blinded to allocation.

The number needed to agree prescription and the confidence in prescription are only reported in studies on individualized (classical) homeopathy, which did not apply to our study. The participants were randomly allocated to receive either Malagueta or placebo, as follows: 5 drops, by mouth, three times a day, for 4 weeks, and then stop taking the medicine for another 4 weeks. Patients and all but one of the researchers (see above) were blinded to allocation. The allocation was at random, as follows: upon enrolment and finishing the first interview, patients were asked to take one flask of medicine from a box. The flasks were randomly numbered, and the allocation list was held by another researcher, not involved with patient recruitment or assessment. The allocation list (simple randomization) was generated on www.sealedenvelope.com.

The Menopausal Vasomotor Symptoms (MVS) questionnaire ${ }^{11}$ was applied at baseline, while the Measure Yourself Medical Outcome Profile (MYMOP) instrument ${ }^{12}$ was applied at baseline and after 1,2,4, and 8 weeks. MYMOP measures the intensity of symptoms (one primary and one secondary), level of activity, and well-being. For this study, the primary symptom was set as hot flashes. A single researcher collected all data. Adherence was monitored by weekly phone calls.

\section{Statistical Analysis}

The primary outcome was the intensity of hot flashes, measured by MYMOP, as above. Secondary outcomes were the secondary symptom on MYMOP, level of activity, and well- being. For sample size calculation, we used a simple approach with Student's $t$ test. The minimal clinically important difference detectable by MYMOP primary symptom is 1.14 points. ${ }^{13}$ In a randomized, double-blinded, placebo-controlled trial that investigated the effects of individualized homeopathy for symptoms of estrogen withdrawal in breast cancer survivors, in which treatment was not superior to placebo, the mean adjusted difference between groups was 0.4 points. $^{14}$

Therefore, we hypothesized that the mean reduction in MYMOP primary symptom should be of at least 1.5 points over that of placebo. Assuming a mean difference between responses of the two groups of 1.5 points in the MYMOP instrument for hot flashes, with a standard deviation of 2.0, power of $80 \%$, and significance of $5 \%$, a total of 30 women would need to be included. Missing values due to voluntary dropouts were treated as worsening to the worst possible outcome, in an intention-to-treat analysis. Results are expressed as count (proportion), median (range), or mean \pm standard deviation, as appropriate. Univariate comparisons between groups at baseline were carried out with Student's $t$ test, Mann-Whitney U test, or chi-square test, as appropriate. The treatment effects for the primary and secondary outcomes were determined by ordinal logistic models, adjusting for repeated measures. We also calculated the mean difference between responses of the two groups and the odds ratio for response to treatment (defined as a reduction of at least three MYMOP categories) for the primary outcome, with corresponding 95\% confidence intervals ( $95 \% \mathrm{CI}$ ), as estimates of effect size. A significance level of 5\% was adopted. Statistical packages Stata (StataCorp LLC; College Station, Texas, USA) and Prism (GraphPad Software; La Jolla, California, USA) were used.

\section{Results}

Between September 2014 and November 2015, 40 women were screened for inclusion criteria and included in the study, 20 in each group (- Fig. 1). Their baseline characteristics are depicted in - Table 1. The two groups were comparable at baseline, except for a slightly younger age in the group receiving Malagueta (non-significantly). There were more dropouts in the control group, although not statistically significantly (6 [30\%] vs 1 [5\%], $p=0.09$ ), all after week 4 .

\section{Treatment Effect}

The effect of Malagueta on the primary outcome, the intensity of hot flashes, assessed by MYMOP, was greater than that of placebo over the 4 weeks of treatment (ordinal logistic model, $p<0.001$, - Fig. 2). Regarding effect size, the mean difference in responses between groups (MYMOP category change) was $0.60 \pm 0.62$ (95\% CI: -0.66 to $1.86 ; p=0.07$ ). The odds ratio for treatment response was 2.78 (95\% CI, 0.77 to 10.05 ; $p=0.20$ ). Of note, hot flashes worsened in both groups after treatment was interrupted (after week 4, data not shown).

MYMOP was also used to assess the intensity of one secondary symptom, chosen by the patients; their level of activity (physical, mental, or social); and their overall well-being. The secondary symptoms the patients chose were tiredness $(n=12)$, insomnia $(n=11)$, irritability $(n=7)$, lack of sexual 


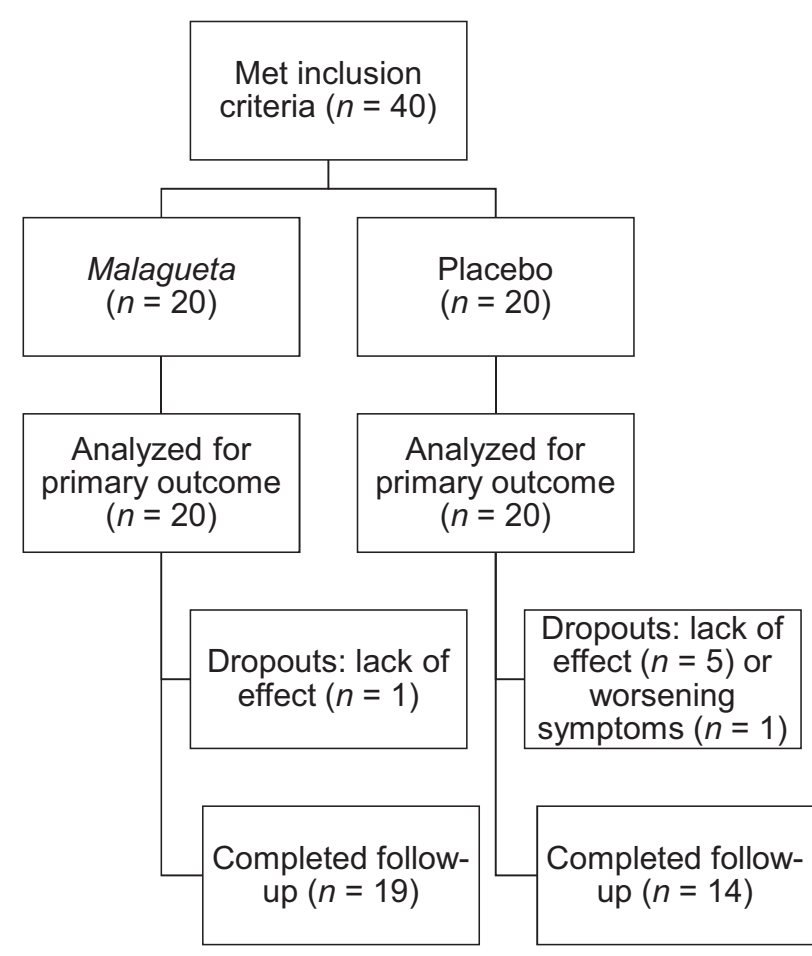

Fig. 1 Flow diagram of study participants. Since all dropouts occurred after week 4 , all enrolled participants were analyzed for the primary outcome ( $n=20$ in both groups). interest $(n=3)$, emotional lability $(n=3)$, sadness $(n=2)$, anxiety $(n=1)$, and none $(n=1)$.

Treatment with Malagueta, compared with placebo, also reduced the intensity of the secondary symptoms (ordinal logistic model, $p=0.001$ ) and improved level of activity $(p=0.025)$ and well-being $(p=0.008)$. No side effects were reported.

\section{Discussion}

We have shown in this study that, in menopausal women, the homeopathic medicine of malagueta pepper, compared with placebo, significantly reduced the intensity of hot flashes, after 4 weeks of treatment. We have also shown that Malagueta ameliorated secondary symptoms, the level of activity, and well-being. The estimates of effect size (mean difference and odds ratio) were not statistically significant. However, the outcome variable (MYMOP) is ordinal and these tests are not adequate for ordinal variables. We therefore opted for the ordinal logistic model as a more robust test for significance in this study.

Hormonal therapy has been the standard treatment for menopause symptoms for many years. However, the risks associated with hormonal therapy have limited its use. Therefore, the number of women who do not want, shall not, or cannot receive hormonal therapy for menopausal hot flashes has been increasing. Thus, a significant proportion of these women have sought alternative therapies, supported by their families and healthcare providers. Since menopause is not a disease in itself, it makes sense that its symptoms are relieved using treatments with the best safety profile. With this in mind,

Table 1 Demographic data and menopausal vasomotor symptoms at baseline

\begin{tabular}{|c|c|c|c|}
\hline & Placebo $(n=20)$ & Malagueta $(n=20)$ & p-Value \\
\hline Age (years) & $52.9 \pm 6.5$ & $49.5 \pm 4.9$ & 0.075 \\
\hline Skin color (white) & $17(85 \%)$ & $12(60 \%)$ & 0.162 \\
\hline Time of amenorrhea (months) & $50(2 ; 244)$ & $13(2 ; 170)$ & 0.192 \\
\hline Body mass index $\left(\mathrm{kg} / \mathrm{m}^{2}\right)$ & $27.3 \pm 3.8$ & $27.5 \pm 4.8$ & 0.891 \\
\hline Previous hormonal therapy & $4(20 \%)$ & $7(35 \%)$ & 0.480 \\
\hline Previous non-hormonal therapy & $6(30 \%)$ & $2(10 \%)$ & 0.235 \\
\hline Duration of previous contraception use (months) & $18(0 ; 240)$ & $36(0 ; 360)$ & 0.828 \\
\hline \multicolumn{4}{|l|}{ Menopausal vasomotor symptoms } \\
\hline Hot flashes for less than 2 years & $8(40 \%)$ & $11(55 \%)$ & 0.527 \\
\hline \multicolumn{4}{|l|}{ Frequency of hot flashes per day } \\
\hline$<5$ & $3(15 \%)$ & $3(15 \%)$ & \multirow[t]{3}{*}{0.151} \\
\hline $5-10$ & $15(75 \%)$ & $10(50 \%)$ & \\
\hline$>10$ & $2(10 \%)$ & $7(35 \%)$ & \\
\hline Hot flashes in daytime (versus at night time) & $9(45 \%)$ & $11(55 \%)$ & 0.527 \\
\hline Duration of hot flashes ( $>5$ minutes) & $5(25 \%)$ & $3(15 \%)$ & 0.429 \\
\hline Sweat during hot flashes (intense) & $9(45 \%)$ & $10(50 \%)$ & 0.752 \\
\hline Interruption of daily activities by hot flashes & $8(40 \%)$ & 7 (35\%) & 0.744 \\
\hline Interruption of sleep by hot flashes & $16(80 \%)$ & $14(70 \%)$ & 0.465 \\
\hline
\end{tabular}

Note: Values are expressed as mean \pm standard deviation, median (minimum; maximum), or count (proportion). Comparisons between groups used Student's $t$ test, Mann-Whitney $\mathrm{U}$ test or chi-square test, as appropriate. 

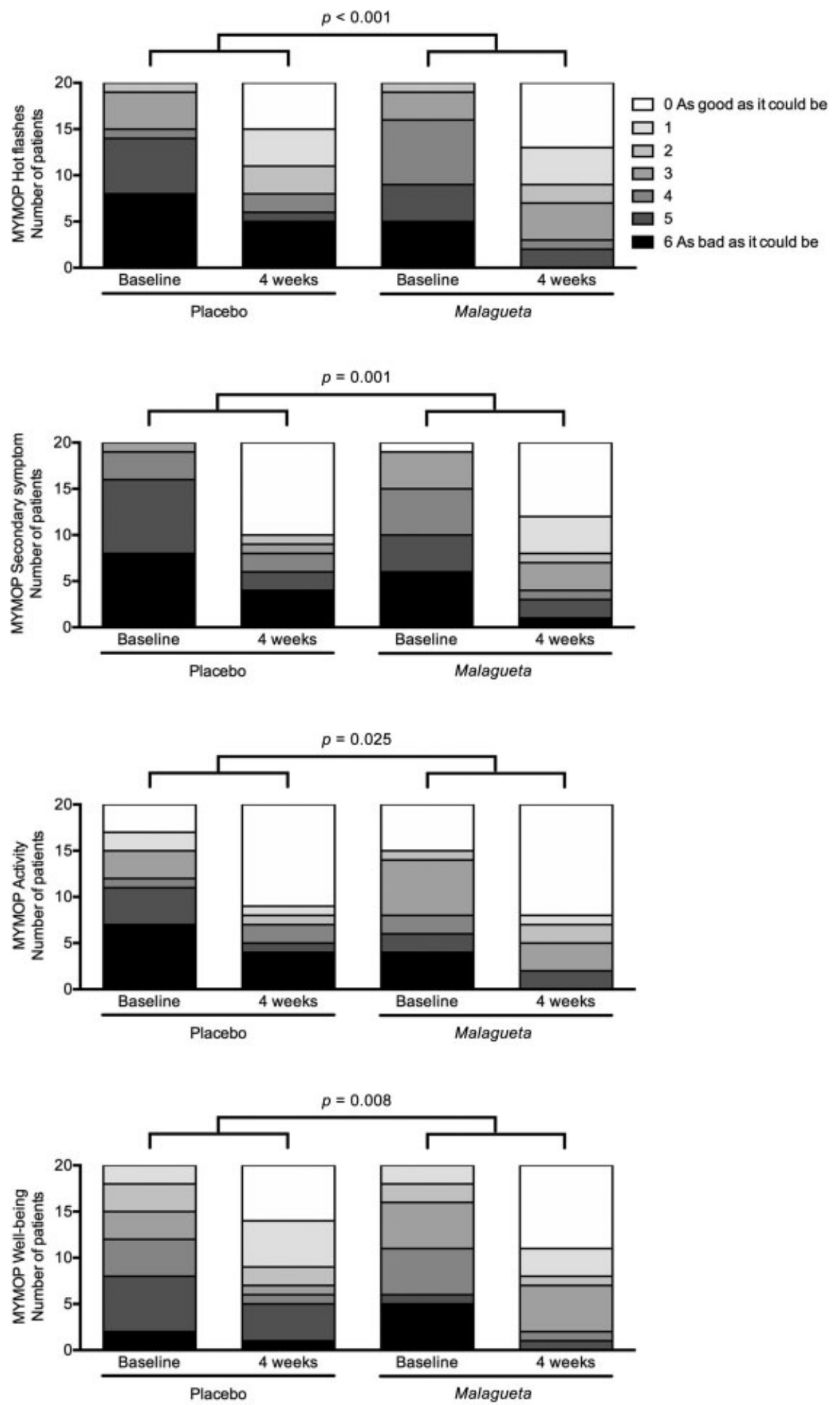

Fig. 2 Intensity of hot flashes assessed by Measure Yourself Medical Outcome Profile (MYMOP) instrument, by treatment and time. The treatment with Malagueta was effective for reducing the intensity of hot flashes $(p<0.001)$ and secondary symptoms $(p=0.001)$, and the level of activity $(p=0.025)$ and well-being $(p=0.008)$, when compared with placebo, as seen by changes in the distribution of patients within ordinal categories.

homeopathic medicines do not have contraindications or lead to severe adverse events; they are also cheap and widely available.

Recently, a review showed that, in observational studies, homeopathic treatments ameliorated hot flashes, fatigue, anxiety, depression, and quality of life in women after menopause and in survivors from breast cancer. ${ }^{3}$ Another underpowered, double-blind, placebo-controlled clinical trial, involving breast cancer survivors, also failed to demonstrate a significant effect of homeopathy on the severity of hot flashes. ${ }^{15}$ These findings reinforce the need for well- 
designed, adequately powered studies to determine whether homeopathy is effective for hot flashes.

Our results are important because Malagueta is the first homeopathic medicine to be effective for hot flashes in a clinical trial. In addition, it is a cheap and available source worldwide, making it an excellent alternative for women seeking relief of hot flashes.

Red peppers are safe for human consumption, both as food and medicine. ${ }^{16-18}$ Capsaicin, among other capsaicinoids, is present in red peppers and is responsible not only for the irritant and burning-like effects but also for a therapeutic effect as a topical analgesic and anti-inflammatory. ${ }^{16}$ Capsaicin also acts on the nervous, cardiovascular, respiratory and gastrointestinal systems, with vasodilatory properties. ${ }^{16-18}$

This study has some limitations. First, a pathogenetic trial of malagueta pepper was not conducted before the clinical trial. However, we believe it was not necessary for our study since the effects of eating peppers in healthy volunteers are very well known. Second, the study had dropouts: overall, there were seven dropouts, six in the placebo group. The reasons the participants gave for dropping out of the study were lack of effect in six patients and one case with worsening of hot flashes. This latter case was not considered an aggravation because she was receiving placebo. Nevertheless, since all dropouts occurred after week 4 , the primary outcome was assessed and analyzed in all patients. There were no losses to follow-up. Therefore, we do not believe there were other reasons for dropping out. Third, this is a phase-2 study and, although we reached the estimated sample size, it involves rather few participants and, therefore, our results need to be confirmed by a larger, phase-3 clinical trial.

\section{Conclusion}

The homeopathic medicine of $C$. frutescens (Malagueta) was significantly superior to placebo in reducing the intensity of hot flashes in menopausal women after 4 weeks of treatment.

\section{Highlights}

- Ingestion of hot peppers (Capsicum frutescens L.) produces symptoms similar to those of menopausal hot flashes.

- A homeopathic medicine was made from fruits of $C$. frutescens, and called Malagueta.

- Malagueta was superior to placebo in alleviating menopausal hot flashes in women after 4 weeks of treatment.

- No side-effects were recorded.

Supplementary Table 1: CONSORT 2010 checklist Supplementary Table 2: RedHot checklist
Funding

None to declare.

Conflict of Interest

All authors declare no conflict of interest.

\section{References}

1 Kronenberg F. Menopausal hot flashes: a review of physiology and biosociocultural perspective on methods of assessment. J Nutr 2010;140:1380S-1385S

2 Sclowitz IK, Santos IS, Silveira MF. Prevalence and factors associated with hot flashes in climacteric and post-climacteric women [article in Portuguese]. Cad Saude Publica 2005;21:469-481

3 Thompson EA. Alternative and complementary therapies for the menopause: a homeopathic approach. Maturitas 2010;66: 350-354

4 Rada G, Capurro D, Pantoja T, et al. Non-hormonal interventions for hot flushes in women with a history of breast cancer. Cochrane Database Syst Rev 2010:CD004923

5 Tropicos [Internet]. Capsicum frutescens L. Available at: http:// www.tropicos.org/Name/296026382018. Accessed April 18, 2018

6 Reifschneider FJB. Capsicum: Hot and Bell Peppers in Brazil [Portuguese]. Brasília: Embrapa Hortaliças; 2000

7 Schulz KF, Altman DG, Moher D; CONSORT Group. CONSORT 2010 statement: updated guidelines for reporting parallel group randomized trials. Ann Intern Med 2010;152:726-732

8 Dean ME, Coulter MK, Fisher P, Jobst K, Walach H. Reporting data on homeopathic treatments (RedHot): a supplement to CONSORT. Homeopathy 2007;96:42-45

9 Harlow SD, Gass M, Hall JE, et al; STRAW 10 Collaborative Group. Executive summary of the Stages of Reproductive Aging Workshop +10 : addressing the unfinished agenda of staging reproductive aging. Menopause 2012;19:387-395

10 BRAZIL. Brazilian Homeopathic Pharmacopea [Portuguese]. 3rd ed. Brazilian Health Regulatory Agency (ANVISA); Brasilia; 2011

11 Ratka A, Miller V, Brown K, et al. Menopausal Vasomotor Symptoms (MVS) survey for assessment of hot flashes. J Womens Health (Larchmt) 2006;15:77-89

12 Paterson C, Britten N. In pursuit of patient-centred outcomes: a qualitative evaluation of the 'Measure Yourself Medical Outcome Profile'. J Health Serv Res Policy 2000;5:27-36

13 Paterson C. Measuring outcomes in primary care: a patient generated measure, MYMOP, compared with the SF-36 health survey. BMJ 1996;312:1016-1020

14 Thompson EA, Montgomery A, Douglas D, Reilly D. A pilot, randomized, double-blinded, placebo-controlled trial of individualized homeopathy for symptoms of estrogen withdrawal in breast-cancer survivors. J Altern Complement Med 2005; $11: 13-20$

15 Jacobs J, Herman P, Heron K, Olsen S, Vaughters L. Homeopathy for menopausal symptoms in breast cancer survivors: a preliminary randomized controlled trial. J Altern Complement Med 2005; $11: 21-27$

16 Newall CA, Anderson LA, Phillipson JD. Herbal Medicines: A Guide for Health-Care Professionals. London: The Pharmaceutical Press; 1996

17 Willoughby MJ, Mills SY. British Herbal Pharmacopoeia. London: British Herbal Medicine Association; 1996

18 Blumenthal M. The Complete German Commission E Monographs: Therapeutic Guide to Herbal Medicines. 1st ed. Massachusetts: American Botanical Council; 1998 